\title{
Dielectric Response of Polyaniline Derivatives and its Effect on the Absorption Spectrum of Modified Copper Phathlocyanine
}

\author{
Khalil J. Hamam ${ }^{1}$ \\ ${ }^{1}$ Applied Physics Department, Tafila Technical University, Tafila, Jordan \\ Correspondence: Khalil J. Hamam, Applied Physics Department, Faculty of Science, Tafila Technical University, \\ P. O. Box: 179, Tafila 66110, Jordan. E-mail: hamam73@yahoo.com
}

Received: July 9, $2014 \quad$ Accepted: July 29, $2014 \quad$ Online Published: October 19, 2014

doi:10.5539/mas.v8n6p123 URL: http://dx.doi.org/10.5539/mas.v8n6p123

\begin{abstract}
The dielectric constant of polyaniline (PANI) derivatives, were investigated over the frequency range from $20 \mathrm{~Hz}$ to $1 \mathrm{MHz}$. The dielectric constant for PANI-base and PANI-salt pellets was decreasing as the frequency increasing. The PANI-salt dielectric constant recorded a huge value of $>10^{8}$ at $20 \mathrm{~Hz}$, and $10^{4}$ at $1 \mathrm{MHz}$. The PANI-base dielectric constant recorded $\sim 11$ at $20 \mathrm{~Hz}$ and $\sim 3.5$ at $1 \mathrm{MHz}$. Their dielectric loss, dielectric loss tangent and ac conductivity were investigated as well. Optical absorption for PANI derivatives in a solution and thin film states were studied. The effect of the PANI-salt's dielectric property in solution and thin film states on the absorption spectrum of modified copper phathlocyanine with sulfonic acid group (CuPc_TS) has been studied. We observed an enhancement of the absorption spectrum of the CuPc_TS to become more broadness and reached the red region of the spectrum compared to the absorption spectrum of CuPc_TS alone. We attributed the more broadness of the CuPc_TS spectrum to the improvement of the CuPc_TS relative permittivity.
\end{abstract}

Keywords: polyaniline, dielectric constant, copper phathlocyanine

\section{Introduction}

Conducting polymers gets scholars attention during the last two decades, due to their electrical, electrochemical, and good thermal and chemical stability. (Dong, 2004; Wei, 1996 \& Roy, 1999) Polyaniline (PANI) is the most interesting type of conducting polymers, they have been used in many applications such as anti-corrosion protection material, (Wang Y., 2004) solar cells, (Wang, W., 2008), and sensors (Ambrosi, 2008). The two famous derivatives types of polyaniline are the emeraldine base (half oxidation state) (PANI-base) and emeraldine salt (full oxidation state) (PANI-salt). One of the most useful types of the polyaniline derivatives is the polyaniline- emeraldine base due its stability at ambient environment (Mirmohseni, 2008). The polymer backbone of the emeraldine base can be protonated with strong acid to produce emeraldine salt leading to an increase in conductivity due to the effect of charge transfer in the polymer backbone (Khan, 2008). In general polyaniline is insoluble in water; polyaniline salt is soluble in strong acids like sulphuric acid and hydrochloric acid (Wang Y., 2004). During the last two decades, many scholars developed a water-soluble Polyaniline (Roy, 1999, Ambrosi, 2008). The conductivity of polyaniline is referred to the polaron and bi-polaron movement of charge carriers along the polymer chain upon applying an electric field; the concentration and mobility of the charge carriers determine the electrical conductivity (Khan, 2008).

Organic materials with high dielectric constant have a potential in many applications, such as electro-active polymers for actuators (artificial muscles) and capacitors industry (X. Zhang et. al, 2005; Bar-Cohen, 2004; Q. Zhang, et. al., 2002; Huang et. al., 2004; Saha, 2010). Dielectric constant (capacitance), dielectric loss and ac conductivity of PANI thin films in frequency range of $100 \mathrm{~Hz}$ to $1 \mathrm{MHz}$ were studied by (Mathai et.al., 2002) which created using A. C. plasma polymerization technique, the dielectric constant values were small $\sim 3$ at 1 MHz. They found that dielectric constant and dielectric loss decreased with frequency and increased with temperature.

The dielectric constant or the relative permittivity is one of the most important electrical properties of materials especially for dielectrics, which is generally defined as $\varepsilon=C / C_{o}$ where $C$ is the capacity of the capacitor (like parallel plates capacitor) when the dielectric material is filled between its plates and $\mathrm{C}_{\mathrm{o}}$ is the capacity when there is no material between its plates. 
The general formula for dielectric constant is given by

$$
\varepsilon^{*}(f)=\varepsilon^{\prime}(f)-\mathrm{i} \varepsilon^{\prime \prime}(f)
$$

where $\varepsilon^{\prime}, \varepsilon^{\prime \prime}$ are the real part of dielectric constant, the imaginary part of the dielectric constant (dielectric loss) which is measure the loss of conductivity in the material at the different frequencies $(f)$ of the electric field.

The dielectric constant depends strongly on the frequency of the electric field (equation 1) or the rate of the change of the time-varying field. So as the frequency increase the time needed for the diploe (assuming that each atom or molecule produces one dipole) to reorient itself with the field direction decreases, so that in general, the dielectric constant decreases as the frequency increases. There are many microscopic polarization mechanisms in materials which depend on the time variation of the electric field such as space-charge (interfacial) which is due the charge movement between materials and electrodes, orientaional polarization which occurs in molecules and materials with permanent dipole moment, ionic polarization which is the distortion of the lattice vibration that's why it's called vibrational polarization and electronic polarization (optical polarization) which is the deformation of the electronic cloud around the positive center of the atom. The electric dipole moment can be induced by the electric field in non-polar materials or be permanent as in polar molecules (Feynman, 1996; Kao, 2004).

In this paper, a dielectric response of polyaniline derivatives was studied for the PANI-base and PANI-salt as pellets. Optical absorption behavior for PANI-salt in solution and thin film states were studied too. Mixing PANI with organic semiconductor solution like copper phathlocyanine $(\mathrm{CuPc})$ could improve the absorption band width of $\mathrm{CuPc}$. $\mathrm{CuPc}$ is the most famous organic semiconductor material which got the scholars attention due to its chemical and thermal stability (Simon, 1985), and its potential in many electronic applications such as organic photovoltaics and light emitting diodes.(Peumans, 2003; Hamann, 1984; Hor, 1983). CuPc is not soluble in regular organic solvents, except in strong acids like sulphuric acid. Solutions of CuPc or PANI in sulfonic acids are not a practical choice in electronic fabrication due to its corrosion effect. So a modified CuPc by a sulphunated acid group such as copper phthalocyanine 3, 4', 4", 4"'-tetrasulfonic acid tetra sodium salt (CuPc_TS), is getting attention in applications require solution process rather than regular physical vapor deposition (thermal evaporation) due its water solubility. It has been used in organic photovoltaic OPV's devices as a donor material or as a replacement of the hole transport layer (HTL) PEDOT: PSS (Bechara, 2012; Schumann, 2011; Ryan, 2012), as a dye (Wohrle, 1989; Thomas, 1990) and as an antiviral drug (against HIV) (Vzorov, 2003).

We are trying to use the PANI-salt dielectric property for improving the CuPc_TS absorption spectrum by changing the semiconductor dielectric constant medium, or the backbone or the energy levels of the polymer. Increasing the dielectric constant of the medium could decrease the columbic attraction between hole-electron pairs (which known as excitons); or changing the energy levels (HOMO-LUMO) of the polymer, could improve of OPV's efficiency.

\section{Experimental}

PANI-salt and PANI-base powders were purchased from Alfa-Aesar and used as received. A grounded powder was pressed by a hydraulic press at room temperature into pellets under $\sim 50 \mathrm{MPa}$. Samples were sandwiched between two copper electrodes; the pellets had the same radius as the electrodes which are $6 \mathrm{~mm}$ in a circular disk shape and the thickness were between $0.75-0.80 \mathrm{~mm}$. For the parallel plate capacitor, the formula to calculate the real and imaginary parts of the dielectric constant of the sandwiched material are given by

$$
\begin{gathered}
\mathcal{E}^{\prime}=\frac{\mathrm{C}_{\mathrm{m}} \mathrm{d}}{\varepsilon_{\mathrm{o}} \mathrm{A}} \\
\mathcal{\varepsilon}^{\prime \prime}=\mathcal{\varepsilon}^{\prime} \mathrm{Df}
\end{gathered}
$$

where $\mathrm{C}_{m}$ is the measured capacitance using Quadtech 1920 meter, $\mathrm{A}$ is the electrode's area, $\mathrm{d}$ is the disks thickness, $\varepsilon_{0}=8.85 \times 10^{-12} \mathrm{~F} / \mathrm{m}$ is the vacuum's permittivity and $\mathrm{Df}$ is the dissipation factor which is equal to $\tan \delta$ (loss tangent; $\delta$ is the loss angle); lower dissipation factor is desired for high performance of electronic applications, if the dissipation factor is small the ability of material to keep charge is larger and the performance of the dielectric material is considered to be better. The amplitude of the ac electric signal applied to the samples was $1 \mathrm{v}$.

Absorption spectra for solution and thin film samples were carried out using Lambda35 UV/Vis 
Spectrophotometers from PerkinElmer. 0.2M solution of PANI-base in Dimethyl sulfoxide (DMSO) and 0.1M solution of PANI-salt in ethanol and N-Methyl-2-pyrrolidone (NMP) were sonicated then stirred for three days. $0.2 \mathrm{M} \mathrm{CuPc}$ CTS water solution was prepared and stirred for a week then filtered using $0.45 \mu \mathrm{m}$ filter papers. CHEMAT spin coater was used for thin film fabrication at low speed about $700 \mathrm{rpm}$.

\section{Results and Discussion}

The dielectric constant, dielectric loss, and dielectric loss tangent for compressed pellets of PANI-base were recorded at room temperature as shown in figure $1 \mathrm{a}, \mathrm{b}$ and $\mathrm{c}$ respectively ; the dielectric constant values recorded 11 at low frequencies and decrease as the frequency increase to record $\sim 3.5$ at $1 \mathrm{MHz}$. The peak of $\varepsilon$ ' and $\varepsilon$ " had a small plateau at low frequency until suddenly decreasing, which could be due to the effect of the interfacial polarizability between the electrodes and the material, which could effective up to $10^{3} \mathrm{~Hz}$.

Figure 1d. Shows the conductivity of PANI-base as a function of frequency, the conductivity increase linearly as the frequency increase without dc $\sigma(0)$ plateau at low frequencies, which usually associated with conductivity according to the universal power law $\sigma(\omega)=\sigma(0)+A \omega^{n}$ where $\omega$ is the angular frequency and $\mathrm{n}$ is the frequency exponent which used to understand the type of conduction mechanism in the materials and $A$ is the ac conductivity factor (Kao, 2004; Raju, 2003). n value from the slope of the curve was $0.6(<1)$ supports the hopping conduction (tunneling of charges) as the type of charge transport mechanism inside the material as suggested by Elliot which is a non-Debye relaxation (Elliot, 1987).
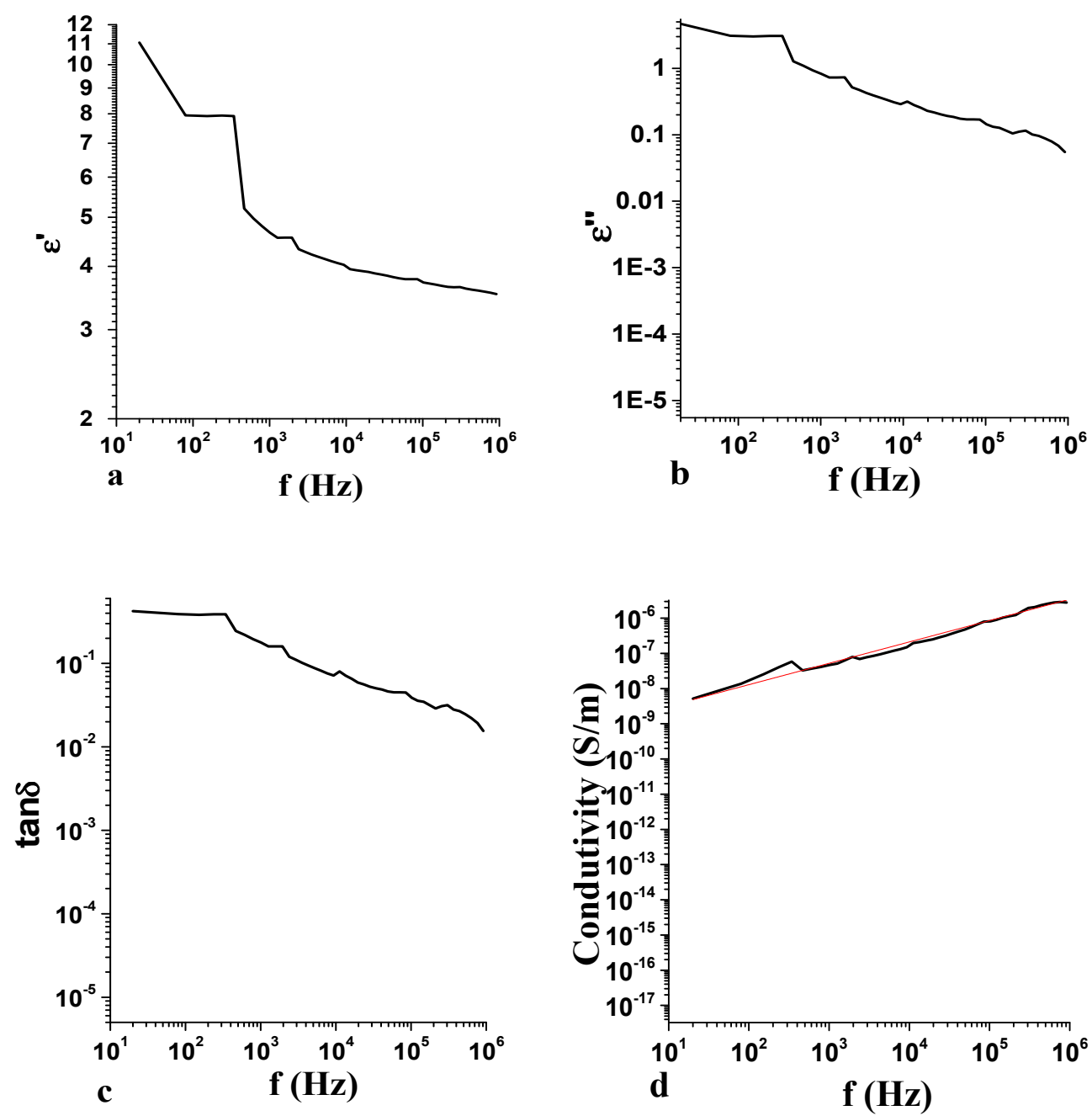

Figure 1. a) Dielectric constant, b) dielectric loss, c) dielectric loss tangent and d) conductivity at room temperature and $25 \% \mathrm{RH}$ for PANI-base

Optical absorption spectrum of PANI-base in DMSO is shown in figure 2, the characteristic bands are Q band $\left(\pi-\pi^{*}\right.$ transition) in the visible region, the peak is located at the wavelength of $628 \mathrm{~nm}$ and B-band (Soret band) 
in the ultraviolet region at $311 \mathrm{~nm}$, where the absorption is more than the Q-band (almost doubled). A small peak is at $871 \mathrm{~nm}$, which is close to the red region.

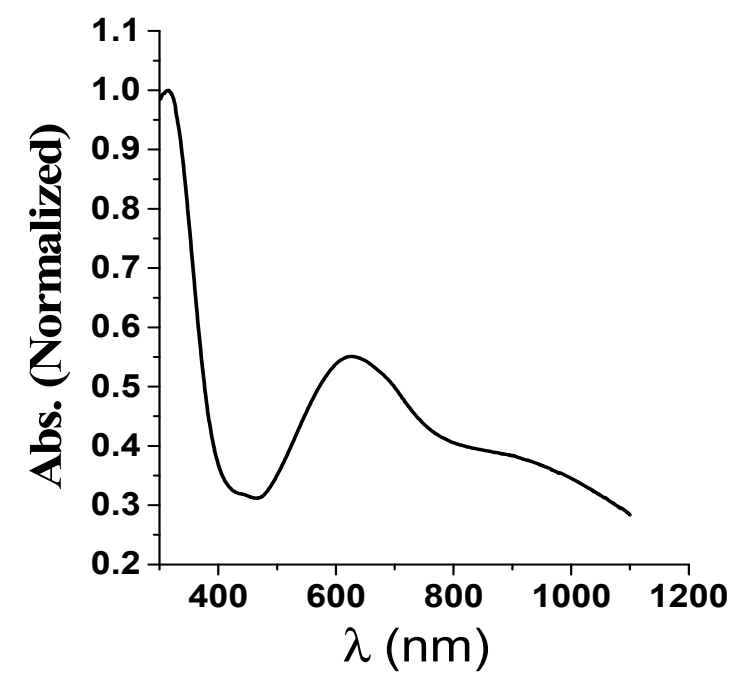

Figure 2. PANI-base absorption spectrum in DMSO solution

The dielectric constant, dielectric loss, dielectric loss tangent and conductivity for compressed pellets of PANIsalt were recorded at room temperature as shown in figure 3. a, b, c and d respectively. The dielectric constant values were very high $>10^{8}$, with a plateau at low-frequency. The dielectric constant decreased almost linearly as the frequency increased to record 4000 at $1 \mathrm{MHz}$. The dielectric loss and loss tangent were high. The high dielectric constant was expected because of the conductive property of the material. $\varepsilon$ ' and $\varepsilon$ " had a plateau at low frequency up to $10^{3} \mathrm{~Hz}$, then eventually decrease linearly until $1 \mathrm{MHz}$. The decrease in $\varepsilon$ ' and $\varepsilon$ " with increasing frequency is attributed to the lags of time between the frequency increasing, and the ability of the molecules to adjust themselves with the direction of the field. Comparing figures $1 \mathrm{c}$ and $1 \mathrm{~d}$ and figure $3 \mathrm{c}$ and $3 \mathrm{~d}$ for tangent loss and conductivity shows almost similar behavior, were the tangent loss decreases, while the conductivity increases with increasing frequency. This can be attributed to Maxwell-Wagner model (space charge model), the model relates the grainy structure and impurities concentration in the material, increasing the frequency indicates a decrease the series resistance, which explain the increase in the conductivity as the frequency increase (Wagner, 1913; Maxwell, 1973).
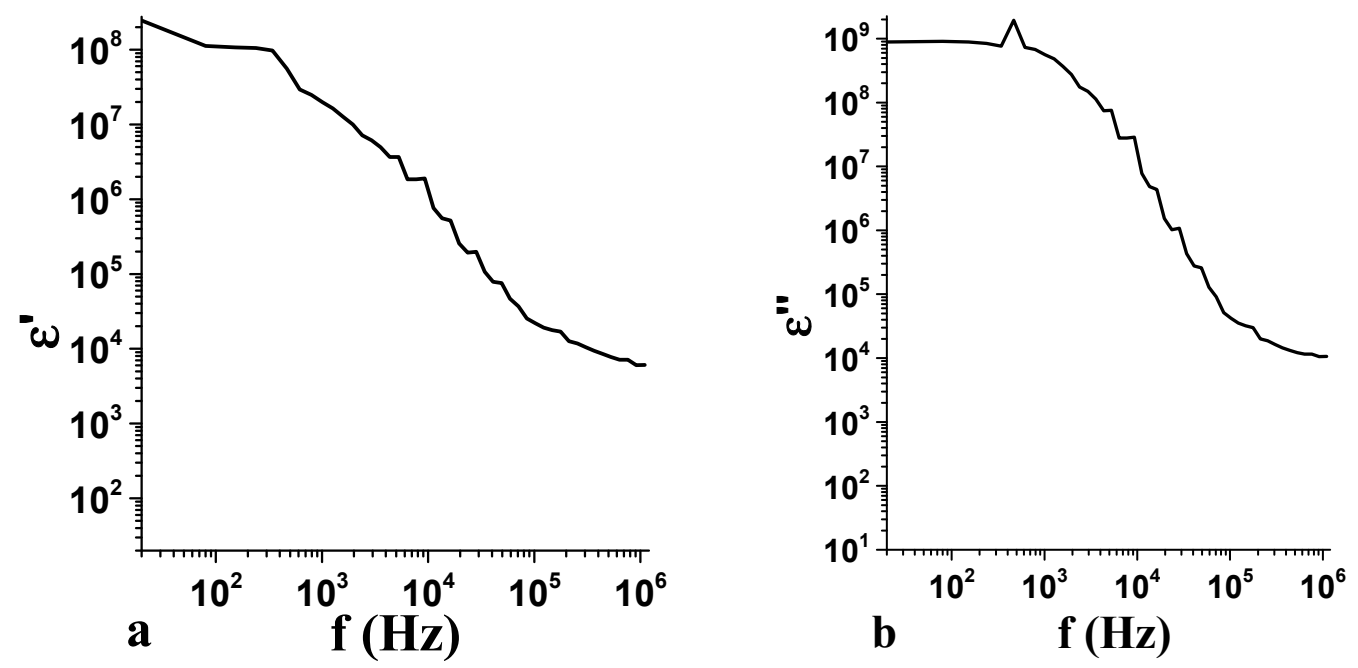

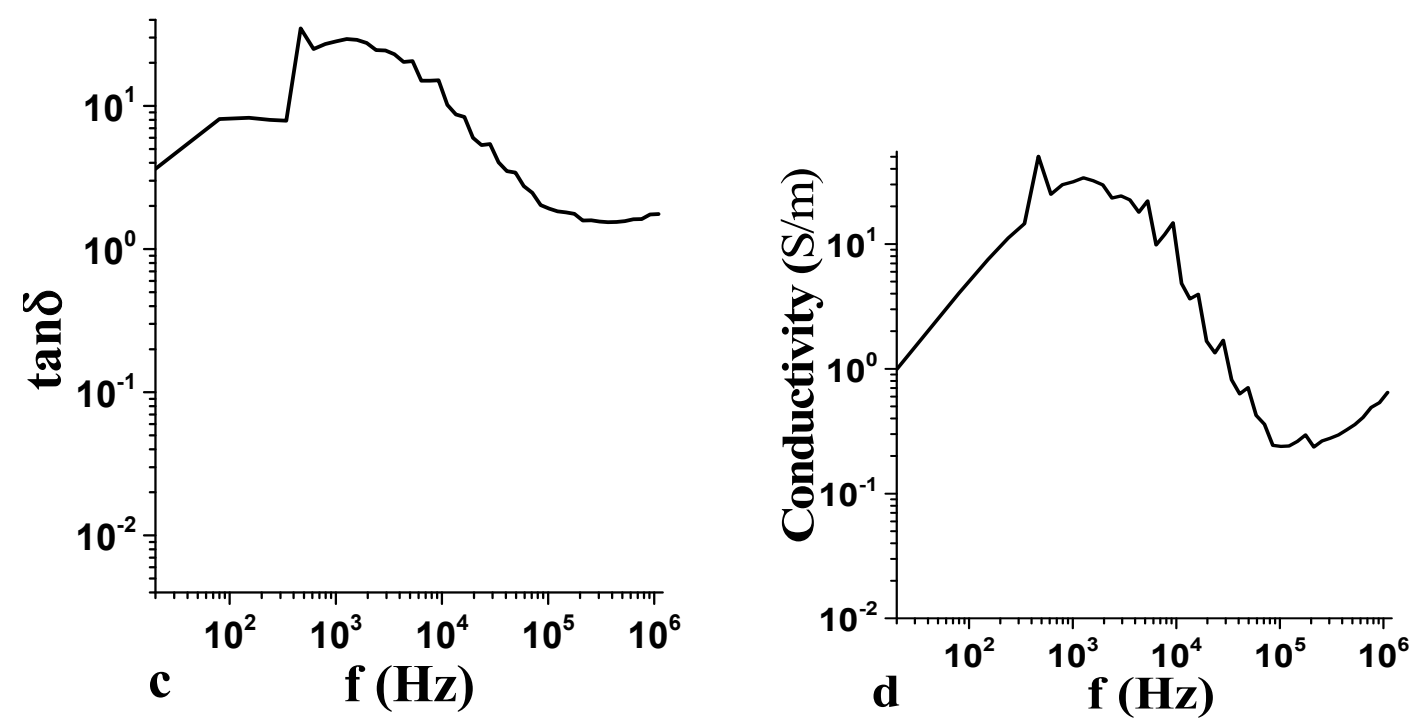

Figure 3. a) Dielectric constant, b) dielectric loss, c) dielectric loss tangent and d) conductivity at room temperature and $25 \% \mathrm{RH}$ for PANI-salt pellets

PANI-salt in ethanol forms a suspension solution. The absorption spectra in ethanol solution and thin film spin coated on Fisher microscope glass were recorded; the suspension forms a nano-size shape with different sizes. The absorption spectrum was broader compared to the PANI-base spectrum in the red and infrared regions "free carrier region" as shown in figure $4 \mathrm{a}$ in ethanol solution and figure $4 \mathrm{~b}$ as a thin film.
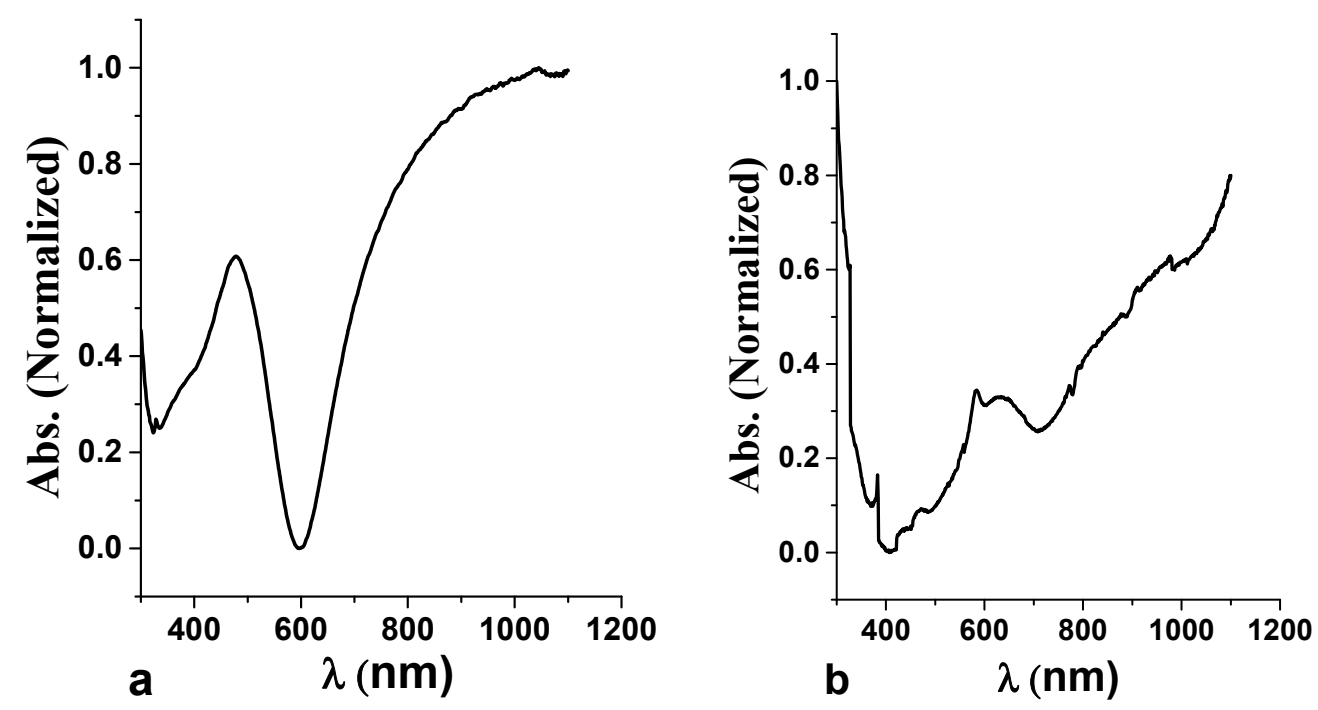

Figure 4. PANI-salt absorption spectrum in ethanol as a) solution and b) thin film (spin coated)

Figure 5a shows the absorption spectrum of CuPc_TS solution in water, where B (333 nm) and Q (611 nm) bands are clearly observed. Adding drops of $0.2 \mathrm{M}$ of CuPc_TS over the PANI-salt and spin coated, improves the intensity of absorption of the PANI-salt spectrum at ultraviolet and visible region as can be observed in figure $5 \mathrm{~b}$ compared to figure 4b. But the small drops didn't affect the broadness of PANI-salt spectra in the IR region as shown in figure $5 \mathrm{~b}$. 

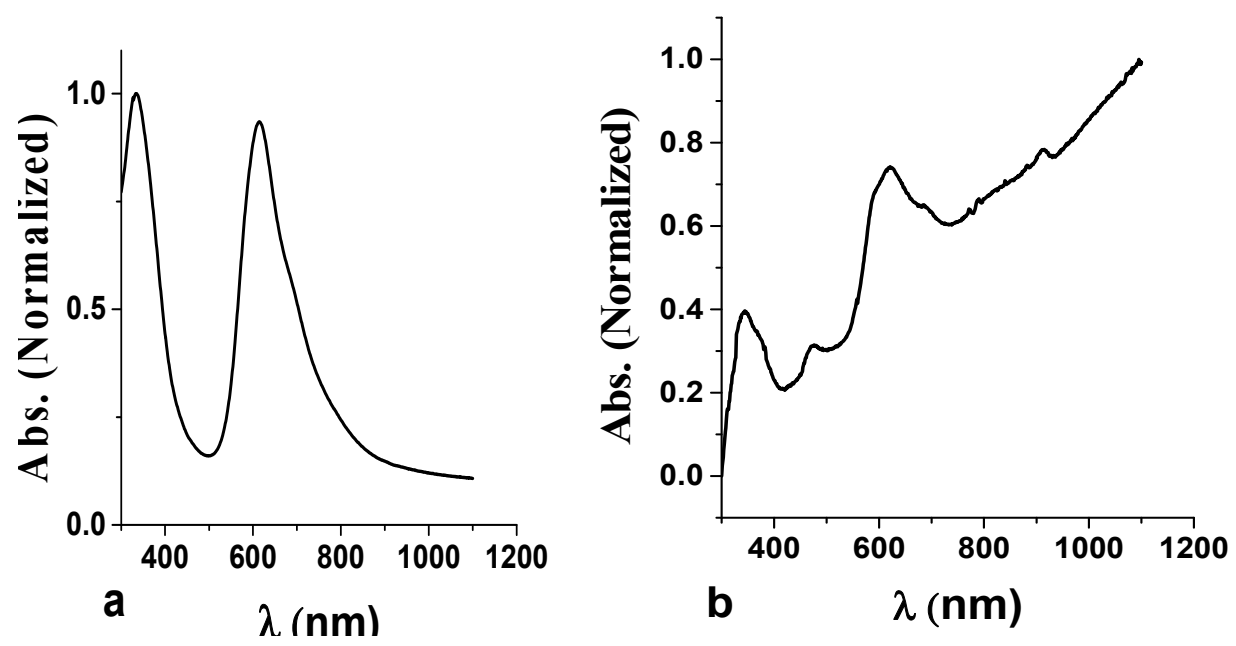

Figure 5. Absorption spectrum of a) CuPc_TS Thin film and b) PANI-salt thin film with CuPc_TS

Solutions of CuPc-TS and PANI-salt due to their miscibility of water and NMP were prepared. The absorption spectrum of spin coated thin films of these solutions is shown in Figure 6; where the effect of PANI-salt on the absorption spectra of the CuPc_TS according to its ratio in the solution. The existence of PANI-salt (2\%) in the solution of CuP_TS as shown in Figure6a leads to create a new peak at $808 \mathrm{~nm}$ wavelength, increasing the PANI-salt percentage to $15 \%$ in the solution increase the intensity at the $808 \mathrm{~nm}$ by $50 \%$ as in figure $6 \mathrm{~b}$. This approach could help to improve the efficiency of organic solar cells based on small molecules such as copper phathlocyanine, which is suffering from low efficiency. The efficiency of organic solar cells is limited due to many factors; one of them is the limited spectral coverage of the solar spectrum, i.e. the absorption of organic semiconductors is narrow and limited to visible region (Forrest, 2005). Further investigation using chemical methods like NMR or basic elemental analysis measurements are required to know the actual reason behind the improvement of the absorption spectrum of CuPc_TS by mixing it with the PANI-salt.
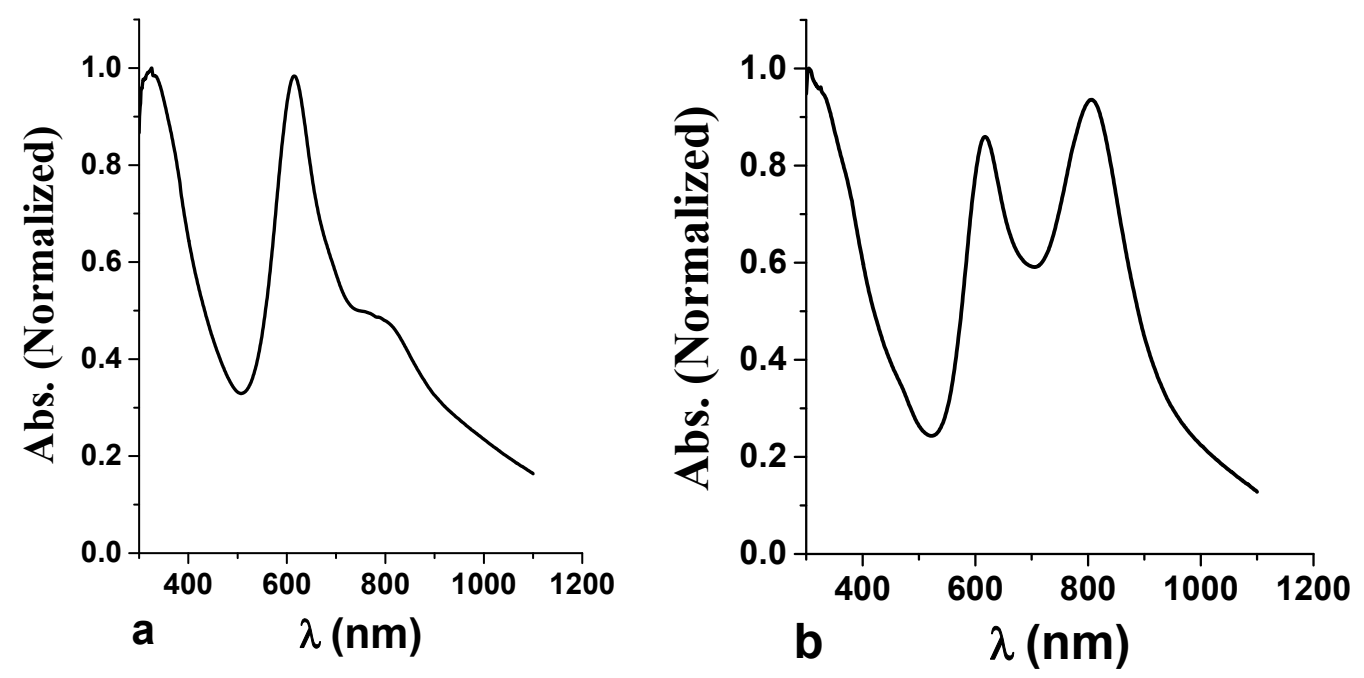

Figure 6. Absorption spectrum of CuPc_TS and PANI_salt Thin films in a different ratios a) $2 \%$ (wt.) and

b) $15 \%$ (wt.)

\section{Conclusion}

The dielectric constant, dielectric loss, dielectric loss tangent and conductivity over frequency range from $20 \mathrm{~Hz}$ to $1 \mathrm{MHz}$ for PANI derivatives were investigated. It was decreasing as the frequency increase. The dielectric 
constant, dielectric loss, dielectric loss tangent and conductivity for PANI-salt were high, which is relevant to the physical property of PANI-salt as a conductive material. The recorded value for the dielectric constant was very high $>10^{8}$ at $20 \mathrm{~Hz}$ and 4000 at $1 \mathrm{MHz}$. The dielectric constant, dielectric loss, dielectric loss tangent and conductivity of PANI-base were very low compared to PANI-salt; the dielectric constant value was $\sim 11$ at $20 \mathrm{~Hz}$ and $\sim 3.5$ at $1 \mathrm{MHz}$, which is expected for the PANI-Base which is considered as a semi-conducting material. The expansion of the CuPc_TS absorption spectra when mixed with PANI-salt could be attributed physically to the improvement of the dielectric constant of the CuPc_TS's medium. Improving the absorption spectrum of CuPc_TS could have a strong influence on the absorption efficiency of organic solar cells based on small molecule materials.

\section{References}

Ambrosi, A., Morrin, A., Smyth, M. R., \& Killard, A. J. (2008). The application of conducting polymer nanoparticle electrodes to the sensing of ascorbic acid. Anal. Chim. Acta. 609, 37-43. http://dx.doi.org/10.1016/j.aca.2007.12.017

Bar-Cohen, Y. (2004) Electroactive Polymers (EAP) Actuators as Artificial Muscles Reality, Potential, and Challenges, 2nd ed., SPIE, Bellingham, WA. http://dx.doi.org/10.1117/3.547465

Bechara, R., Petersen, J., Gernigon, V., Leveque, P., Heiser, T., Toniazzo, V., Ruch, D., \& Michel, M. (2012). PEDOT:PSS-free organic solar cell susing tetrasulfonic copper phthalocyanine as buffer layer. Solar Energy Materials and Solar Cells, 98, 482-485. http://dx.doi.org/10.1016/j.solmat.2011.11.005

Dong, H., Prasad, S., Nyame, V., \& Jones, W. (2004). Sub-micrometer conducting polyaniline tubes prepared from polymer fiber templates. Chem. Mater., 16, 371-373. http://dx.doi.org/10.1021/cm0347180

Elliot, S. R. (1987). AC conduction in amorphous-chalcogenide and pnictide semiconductors. Adv. Phys., 36, 135. http://dx.doi.org/10.1080/00018738700101971

Feynman, R. P., Leighton, R. B., \& Sands, M. (1989). The Feynman Lectures on Physics, Addison-Wesley, Reading, PA, Chap. 11. http://dx.doi.org/10.1002/aic.690100602

Forrest, S. (2005). The Limits to Organic Photovoltaic Cell Efficiency. MRS BULLETIN, 30. $\mathrm{http}: / / \mathrm{dx}$.doi.org/10.1557/mrs2005.5

Hamann, C., Hoehne, H. J., \& Naegler, M. (1984). Parameters of lead phthalocyanine solar cells and their improvement. Mater. Sci., 10, 105.

Hor, A. M., Loutfy, R. O., \& Hsiao, C. K. (1983). Photovoltaic properties of cadmium sulfide/trivalent-metal phthalocyanine heterojunction devices. Appl. Phys. Lett., 42, 165. http://dx.doi.org/10.1063/1.93861

Huang, C., Zhang, Q. M., deBotton, G., \& Bhattacharya, K. (2004). All-organic dielectric-percolative three-component composite materials with high electromechanical response. J. Appl. Phys. Lett., 84, 4391. http://dx.doi.org/10.1063/1.1757632

Kao, K. C. (2004). Dielectric Phenomena in Solids with Emphasis on Physical Concepts of Electronic Processes, Academic Press, London, UK.

Khan, S. N. (2007). Electrospinning Polymer Nanofibers - Electrical and Optical Characterization, Ohio University, Ph.D thesis.

Mathai, C. J., Saravanan, S., Anantharaman, M. R., Venkitachalam, S., \& Jayalekshmi, S. (2002). Characterization of low dielectric constant polyaniline thin film synthesized by ac plasma polymerization technique. J. of Physics D: Applied Physics, 35(3), 240-245. http://dx.doi.org/10.1088/0022-3727/35/3/312

Maxwell, J. C. (1973). Electricity and Magnetism, Oxford University Press.

Mirmohseni, A., \& Houjaghan, M. R. (2008). Synthesisand characterization of water-soluble conducting poly (3-amino-4-methoxybenzenesulfonic acid). Mol. Cryst. Liq. Cryst., 484, 722-727. http://dx.doi.org/10.1080/15421400801904898

Peumans, P., Yakimov, A., \& Forrest, S. R. (2003). Small molecular weight organic thin-film photodetectors and solar cells. J. Appl. Phys., 93, 3693. http://dx.doi.org/10.1063/1.1534621

Raju, G. G. (2003). Dielectrics in electric fields, M. Dekker inc. N.Y.

Roy, B. C., Gupta,M. D., Bhowmik, L., \& Ray, J. K. (1999). Studies on water soluble conducting polymer-Aniline initiated polymerization of m-aminobenzene sulfonic acid. Synth. Met., 100, 233-236. http://dx.doi.org/10.1016/S0379-6779(98)01505-7 
Ryan, J. W., Anaya-Plaza, E., Escosura, Torres, A. T., \& Palomares, E. (2012). Small molecule solar cells based on a series of water-soluble zinc phthalocyanine donors. Chem. Com., 48, 6094-6096. http://dx.doi.org/10.1039/c2cc30676j

Saha, R., \& Mandal, B. K. (2010). New Copper Phthalocyanine Oligomers for High Dielectric Constant Polymer Films. J. Appl. Polym. Sci., 117, 122. http://dx.doi.org/10.1002/app.31749

Schumann, S., Hatton, R. A., \& Jones, T. S. (2011). Organic Photovoltaic Devices Based on Water-Soluble Copper Phthalocyanine. J. Phys. Chem. C, 115, 4916-492. http://dx.doi.org/10.1021/jp109544m

Simon, J., Andre, J. J., Lehn, J. M., \& Rees, C. W. (1985). Molecular Semiconductors: Photoelectrical Properties and Solar Cells, Springer, Berlin. http://dx.doi.org/10.1007/978-3-642-70012-5

Thomas, A. L. (2003). Phthalocyanine Research and Applications, CRC press, U.S.A.

Vzorov, A. N., Marzilli, L. G., Compans, R. W., \& Dixon, D. W. (2003). Prevention of HIV-1 infection by phthalocyanines. Antiviral Res. 59, 99-109. http://dx.doi.org/10.1016/S0166-3542(03)00035-4

Wagner. K. W. (1913). Ann. De. Physik., 40, 817.

Wang, W., Schiff, E., \& Wang, Q. (2008). Amorphous silicon/polyaniline heterojunction solar cells: Fermi levels and open-circuit voltages. J. Non-Crystalline Solids, $354, \quad 2862-2865$. http://dx.doi.org/10.1016/j.jnoncrysol.2007.10.104

Wang, Y. Y., \& Jing, X. L. (2004). Preparation of an epoxy/polyaniline composite coating and its passivation effect on cold rolled steel. Polym. J. 36, 374-379. http://dx.doi.org/10.1295/polymj.36.374

Wei, X. L., Wang, Y. Z., Long, S. M. Bobeczko, C., \& Epsteib, A. J. (1996). Synthesis and physical properties of highly sulfonated polyaniline. J. Am. Chem. Soc., 118, 2545 -2555. http://dx.doi.org/10.1021/ja952277i

Wöhrle, D. (1989). Phthalocyanines: Properties and Applications. Phthalocyanines in Polymer Phases. Inc., 55-132. VCH Publishers, Inc. N.Y.

Zhang, Q. M., Li, H., Poh, M., Xia, F., Xu, Z. Y., Cheng, \& Huang, H. C. (2002). An all-organic composite actuator material with a high dielectric constant. Nature, 419, 284. http://dx.doi.org/10.1038/nature01021

Zhang, X., Löwe, C., Wissler, M., Jähne, \& Kovacs, B. G. (2005). Dielectric elastomers in actuator technology. Advance Engineering Materials, 7, 361. http://dx.doi.org/10.1002/adem.200500066

\section{Copyrights}

Copyright for this article is retained by the author(s), with first publication rights granted to the journal.

This is an open-access article distributed under the terms and conditions of the Creative Commons Attribution license (http://creativecommons.org/licenses/by/3.0/). 